Anita Traninger

\title{
Las ninfas de los ríos: Echo(s) zwischen Miguel de Cervantes, Paul Valéry und Jorge Luis Borges als Grundlegung einer Theorie literarischer Resonanz
}

https://doi.org/10.1515/arcadia-2021-9015

Abstract: The article takes as its starting point a reading of Jorge Luis Borges' short story Pierre Menard, autor del Quijote, focusing on the first passage from Cervantes' Quijote that is quoted verbatim in the text. An invocation of the river nymphs and the nymph Echo („las ninfas de los ríos, la húmida y dolorosa Eco“), it is singled out by the narrator as bearing the voice of Pierre Menard despite having never been attempted by him in his project of writing Don Quijote again. I argue that the invocation of Echo does not point to a duplication of the text. Rather, Echo's early modern acceptation, that of a dialogue partner that not only answers, but answers back and says different things with the same words, encapsulates Menard's project as such and, beyond that, a theory of literary resonance. Paul Valery's poems and essays, to which Borges' story variously alludes, underpin this reading of Echo as the patron saint of a theory of resonance that accounts for the necessary openness of literary texts to deviant interpretations, in particular those that could not have been foreseen or desired by their authors.

Keywords: echo, resonance, reader, literary history

Beau ciel, vrai ciel, regarde-moi qui change !

(Paul Valéry, Le Cimetière marin)

„Las ninfas de los ríos“ - dieses Zitat stammt aus einer der bekanntesten Erzählungen von Jorge Luis Borges, „Pierre Menard, autor del Quijote“, und es stammt zugleich aus Miguel de Cervantes' El ingenioso hidalgo Don Quijote de la Mancha. Der erstgenannte Text ist zuerst im Mai 1939 in der argentinischen Zeitschrift Sur publiziert worden, um dann 1941 in der Sammlung El jardín de senderos que se bifurcan (Der Garten der Pfade, die sich verzweigen) wieder zu erscheinen, die

\footnotetext{
Kontaktperson: Anita Traninger, Freie Universität Berlin, Institut für Romanische Philologie, Habelschwerdter Allee 45, 14195 Berlin, Deutschland, E-Mail: anita.traninger@fu-berlin.de 
wiederum 1944 ihren Weg in den Band Ficciones fand. Der Don Quijote, 1605 erstmals im Druck erschienen, war ein instantan multiplizierter Text, und zwar nicht nur, weil er sich als Übersetzung eines arabischen Manuskripts inszeniert, sondern weil er noch im Jahr seines Erscheinens trotz des Druckerprivilegs Juan de la Cuestas in Lissabon und Valencia raubgedruckt wurde. Ein zweiter Teil wurde schließlich zuerst 1614 von Alonso Fernández de Avellaneda und dann 1615 von Miguel de Cervantes veröffentlicht.

Borges' Erzählung handelt von einer weiteren, auf den ersten Blick weitaus unwahrscheinlicheren Verdopplung. Die Erzählung geriert sich als Nachruf und, eingangs, zugleich als Werkkatalog. Pierre Menard, ein französischer Dichter des frühen 20. Jahrhunderts, sei soeben zu Grabe getragen worden, sein Andenken freilich erscheint bereits gefährdet und müsse dringend gesichert bzw. sogar schon korrigiert werden - dies der fiktive Erzählanlass. Menard habe nicht nur einen beachtlichen Katalog publizierter und unpublizierter Werke hinterlassen - der getreulich als bibliographie raisonnée gelistet wird; es gebe auch, so der Erzähler, der sich als enger Freund darstellt, ein unsichtbares, unterirdisches Werk: Pierre Menard hatte nicht weniger als den Don Quijote nochmals schreiben wollen. Nicht abschreiben, nicht imitieren, sondern wortgleich aus sich selbst heraus nochmals schreiben:

No quería componer otro Quijote - lo cual es fácil - sino el Quijote. Inútil agregar que no encaró nunca una transcripción mecánica del original; no se proponía copiarlo. Su admirable ambición era producir unas páginas que coincidieran - palabra por palabra y línea por línea - con las de Miguel de Cervantes. („Pierre Menard, autor del Quijote“ 446)

Er wollte nicht einen anderen Quijote verfassen - was leicht ist -, sondern den Quijote. Unnütz hinzuzufügen, daß er niemals eine mechanische Transkription des Originals ins Auge faßte; er wollte es nicht kopieren. Sein bewundernswerter Ehrgeiz war es, ein paar Seiten hervorzubringen, die - Wort für Wort und Zeile für Zeile - mit denen von Miguel de Cervantes übereinstimmen sollten. („Pierre Menard, Autor des Quijote“ 39)

Gediehen ist das Projekt nicht besonders weit: was Menard hinterlassen hat, sind die Kapitel 9 und 38 sowie ein Fragment des 22. Kapitels des ersten Teils („Pierre Menard, autor del Quijote“ 446). Menards Text, diesen Nachweis versucht der Erzähler zu führen, übertreffe den Quijote des Cervantes im Hinblick auf seine Komplexität:

El texto de Cervantes y el de Menard son verbalmente idénticos pero el segundo es casi infinitamente más rico. (Más ambiguo dirán sus detractores; pero la ambigüedad es una riqueza.) (449)

Der Text von Cervantes und der Text von Menard sind Wort für Wort identisch, aber der zweite ist nahezu unendlich viel reicher. (Zweideutiger, werden seine Verlästerer sagen; aber die Zweideutigkeit ist ein Reichtum.) (43) 
Der Erzähler unserer Geschichte kann sich dem Sog der neuen Interpretationsdimension nicht entziehen - er sieht nicht in Menards Version ein Echo des vorgängigen Cervantes, sondern umgekehrt, im Text des Cervantes findet er ein Echo Menards. Die Konventionen der definitionsgemäß monodirektionalen Rezeptionsbeziehung sind auf den ersten Blick absurd invertiert:

Confesaré que suelo imaginar que la terminó y que leo el Quijote - todo el Quijote - como si lo hubiera pensado Menard? Noches pasadas, al hojear el capítulo XXVI - no ensayado nunca por él - reconocí el estilo de nuestro amigo y como su voz en esta frase excepcional: las ninfas de los ríos, la dolorosa y húmida Eco. (447)

Soll ich gestehen, daß ich mir vorzustellen pflege, er hätte es vollendet und daß ich den Quijote - den ganzen Quijote - so lese, als hätte Menard ihn erdacht? Als ich vor ein paar Abenden im Kapitel 26 blätterte - das er nie in Angriff genommen hat -, erkannte ich den Stil unseres Freundes und beinahe seine Stimme in diesem außergewöhnlichen Satz: Las ninfas de los ríos, la dolorosa y húmida eco [,Die Nymphen der Flüsse, die schmerzensreiche und feuchte Echo']. (40)

„Pierre Menard, autor del Quijote“ ist als ein Manifest der Geburt des Lesers gelesen worden, als Vorwegnahme von Rezeptionsästhetik und Poststrukturalismus gleichermaßen (vgl. Jauß, Theorie der Rezeption 30-35). Er sagt uns aber, und das will ich im Folgenden entfalten, zugleich etwas über Texte selbst, und zwar darüber, wie sie durch die Zeit nicht mit sich selbst identisch bleiben bzw. bleiben können. Natürlich ist es kein Zufall, und es wurde in der Forschung auch bereits registriert, dass der Erzähler ein Echo Menards in einer Passage über die Nymphe Echo hört (Dopico Black 35-36). Ich werde über die vorliegenden Befunde hinausgehend zeigen, dass die Anrufung der Echo auf mehr und anderes verweist als auf die Verdopplung des Textes - denn erstaunlicherweise wurde die Semantik der Nymphe in der ausufernden Borges-Forschung bisher nicht entsprechend in den Blick genommen.

Betrachten wir zunächst den Kontext der entsprechenden Passage im Don Quijote näher. Das 25. Kapitel des ersten Teils führt Quijote und Sancho in die Wildnis der Sierra Morena. Während Sancho weiterziehen will, weckt die Einöde bei Quijote die Erinnerung an Episoden in seinen Ritterbüchern. Er will es dem Amadís de Gaula gleichtun, dem Helden des wirkmächtigsten Ritterromans der Iberischen Halbinsel. Dieser Prosaroman war auf der Grundlage einer langen mündlichen Tradition von Garci Rodríguez de Montalvo verfasst und 1508 erstmals in den Druck gebracht worden. Die Szene, an die sich Quijote erinnert fühlt, ist jene, als sich Amadís auf den sogenannten Armutsfelsen, die Peña Pobre, zurückzieht, nachdem ihn seine verehrte Dame Oriana zurückgewiesen hat. Quijote befindet, dass diese Art von Nachahmung ihm leichter fallen würde als die bis dahin recht erfolglosen Kämpfe gegen Riesen und andere Bösewichte. 
Sanchos Einwand, dass Quijote anders als sein Vorbild gar keinen Grund zu so einer Liebesbuße habe, lässt dieser naturgemäß nicht gelten: Die wahre Probe sei, ohne Anlass wahnsinnig zu werden (,desatinar sin occasión“ 304). Das ist eine Meditation über imitatio, welche freilich in Quijotes ver-rückter Welt nicht im Schreiben mündet, sondern Handeln provoziert. Sie hängt nicht von individuellem Erleben ab, vielmehr suppliert die Tradition voraufgegangener Texte die Verfahrensmuster zusammen mit dem je zugehörigen Affektregime.

Doch zurück in die Diegese: Über ihren Diskussionen waren Quijote und Sancho dahingeritten und finden sich plötzlich an einem locus amoenus wieder. Quijote vermutet sofort Nymphenpräsenz und fällt ansatzlos in den Modus des liebeskranken Schäfers:

¡Oh vosotras, napeas y dríadas, que tenéis por costumbre de habitar en las espesuras de los montes, así los ligeros y lascivos sátiros, de quien sois, aunque en vano, amadas, no perturben jamás vuestro dulce sosiego, que me ayudéis a lamentar mi desventura, o, a lo menos, no os canséis de oílla! (Cervantes 307)

O ihr Oreaden und Dryaden, die ihr in der Berge Dickicht weilt, mögen die leichtfüßigen, lüsternen Satyrn, die auch vergeblich begehren, niemals eure süße Ruhe stören: helft mir, mein Unheil zu beklagen, oder hört mich zumindest duldsam an! (Cervantes dt. 253)

Nachdem er Sancho mit einem Brief zu Dulcinea geschickt hat, vertreibt sich Quijote die Zeit damit, Verse in Rinden zu ritzen - so macht man das als verliebter Schäfer, wie Vergils Bucolica lehren (Ecl. 5, 13-15). Der Erzähler bietet uns ein deplorables Bild des Ritters von der traurigen Gestalt, und es ist an dieser Stelle, dass das vermeintliche Menard-Echo sich manifestiert:

En esto [i.e. en escribir], y en suspirar, y en llamar a los faunos y silvanos de aquellos bosques, a las ninfas de los ríos, a la dolorosa y húmida Eco, que le respondiese, consolasen y escuchasen, se entretenía, y en buscar algunas yerbas con que sustentarse en tanto que Sancho volvía. (Cervantes 321, Kursivschrift A. T.)

Mit derlei Dingen, mit Seufzern und dem Anrufen der Faune und Waldgötter, der Flussnymphen, der schmerzensreichen und feuchten Echo, auf dass diese ihm antworte, jene ihn trösteten und anhörten, verbrachte er seine Zeit und sammelte Kräuter, um sich zu ernähren, bis Sancho wiederkäme [...]. (Cervantes dt. 268, Änderungen von A. T.)

Die Passage aus dem Amadís de Gaula, auf die Quijote sich imitativ verpflichtet hat, ist bemerkenswerterweise ganz anders organisiert. Während die Buße durch die Zurückweisung durch Oriana motiviert ist, fehlt die pastorale Szenerie dort völlig. Es ist allerdings diese Landschaft, die Don Quijote in einen bestimmten Diskursmodus versetzt hat, und streng genommen ist das - und nur das - ihre Funktion. Sie fungiert gleichsam als foucauldianisches Dispositiv, indem sie eine 
bestimmte Art zu sprechen und sich zu verhalten bedingt. Sie verwandelt diejenigen, die sie betreten. Das literarische ,Arkadien' - schon lang spricht man in der Frühen Neuzeit nicht mehr von einem Landstrich auf der Peloponnes, wenn von ,Arcadia“ die Rede ist - ist nur auf den ersten Blick eine durch bestimmte ästhetische Qualitäten herausragende Landschaft. Ernst Robert Curtius hat vom ,Topos‘ des locus amoenus gesprochen, der sich durch eine Reihe von Elementen - „sechs Landschaftsreize“, sagt Curtius (203) - auszeichne. Unter Berufung auf Libanios (4. Jh. n.Chr.) stellt Curtius sie vor: „Ursachen des Frohsinns sind Quellen und Pflanzungen und Gärten und sanfte Lüfte und Blumen und Vogelstimmen.“ (203-204) Es sind dies bestimmte, standardisierte Naturaspekte, die sprachlich evoziert werden - mehr noch: die nur in und durch Sprache existieren. Oder, mit der schönen Maxime Karlheinz Stierles gesagt: „Mit Arkadien hat die Dichtung das Bild einer Landschaft gefunden, die tief in das Medium der Sprachlichkeit eingesenkt ist.“ (50) Diese konventionalisierte Beschreibung muss in Texten weder vollständig gegeben noch ekphrastisch detailliert werden. Denn eine wie immer geartete mimetische Ausgestaltung der Kulisse ist nachrangig gegenüber dem generischen Signal, das hier gesetzt wird: Was textuell aus konventionalisierten Versatzstücken montiert wird, ist jene literarische Hirtenwelt, die mehr noch als eine stilisierte Ideallandschaft eine geistige Landschaft ist, wie Bruno Snell formulierte. Sie ist wesentlich ein Transferprodukt, in dem Elemente der antiken Bukolik mit rinascimentalen und humanistischen Anliegen kontaminiert werden.

Am wichtigsten aber: es ist eine Landschaft, die antwortet. Die Flüsse schwellen an, nicht vom Regen (den gibt es hier nicht), sondern von den Tränen der Schäferinnen und Schäfer; die Bäume neigen sich zu den Seufzern der Liebenden, die Vögel begleiten singend das Schluchzen der Verzweifelten. In einem der wirkmächtigsten Schäferromane, Los siete libros de la Diana, die der Portugiese Jorge de Montemayor 1559 vorlegte und damit die sogenannte novela pastoril, den Schäferroman in Prosa, auf der Iberischen Halbinsel begründete, trägt die Schäferin Belisa diesen Gedanken in einer flammenden Rede vor, die das Stilmittel der rhetorischen Frage ausreizt:

¿Quién pensáis que hace crecer la verde yerba desta isla y acrecentar las aguas que la cercan sino mis lágrimas? ¿Quién pensáis que menea los árboles deste hermoso valle sino la vos [sic] de mis sospiros tristes [...]? ¿Por qué pensáis que cantan los dulces pájaros por entre las matas cuando el dorado Phebo está en toda su fuerza, sino para ayudar a llorar mis desventuras? (Montemayor 229-230)

Wer, meint ihr, lässt das grüne Gras dieser Insel wachsen und die Wasser anschwellen, die sie umgeben, wenn nicht meine Tränen? Wer meint ihr, schüttelt die Bäume dieses schönen Tals, wenn nicht der Klang meiner traurigen Seufzer? Warum, meint Ihr, singen die süßen 
Vögel in den Sträuchern, wenn der goldene Phöbus in voller Kraft steht, wenn nicht um mir zu helfen, mein Unglück zu beweinen? (Übersetzung A. T.)

Montemayor hat diese Denkfigur nicht erfunden. Sie geht auf Petrarcas Canzoniere zurück („Valle che de' lamenti miei se' piena, / fiume che spesso del mio pianger cresci“ / 301, V. 1-2; dazu Warning 2003) und wurde nicht nur in seinem Gefolge vielfach bemüht. Die Welt, die Don Quijote betritt, indem er sie anspricht, ist daher ein literarisch mehrfach codierter Resonanzraum, der zudem traditionell bewohnt ist. Quijotes Anrufung der „ninfas de los ríos“ und der „,dolorosa y húmida Eco“, die Borges’ Erzähler für ganz typisch ,Menard“ hält, spricht zwei mythologische Wesen bzw. Gruppen an: die von Ovids Metamorphosen her bekannte Nymphe Echo und das Kollektiv der Flussnymphen. In der spanischen Literatur ist es insbesondere ein Autor, der uns die Idee nahelegte, dass aus den Flüssen in unserer unmittelbaren Umgebung jederzeit Nymphen auftauchen können. Garcilaso de la Vega, der Soldat in Diensten Karls des V., der erst nach seinem Tod 1536 weithin als Dichter bekannt wurde und der gemeinsam mit Juan Boscán die italienischen Metren des Petrarkismus nach Spanien gebracht hatte, dieser Garcilaso evoziert an zwei Stellen in seinem schmalen Euvre die Idee, dass unter der Wasseroberfläche eine magische Anderswelt sich verberge, und dass die darin hausenden Nymphen eine besondere Respondenzaufgabe hätten - in seiner Dritten Ekloge und im 11. Sonett, das hier ausführlich zitiert sei:

\footnotetext{
Hermosas ninfas, que, en el río metidas, contentas habitáis en las moradas de relucientes piedras fabricadas y en columnas de vidrio sostenidas; agora estéis labrando embebecidas, o tejiendo las telas delicadas;

agora unas con otras apartadas, contándoos los amores y las vidas;

dejad un rato la labor, alzando vuestras rubias cabezas a mirarme, y no os detendréis mucho según ando;

que o no podréis de lástima escucharme, o convertido en agua aquí llorando, podréis allá de espacio consolarme. (Garcilaso, Soneto XI)
} 


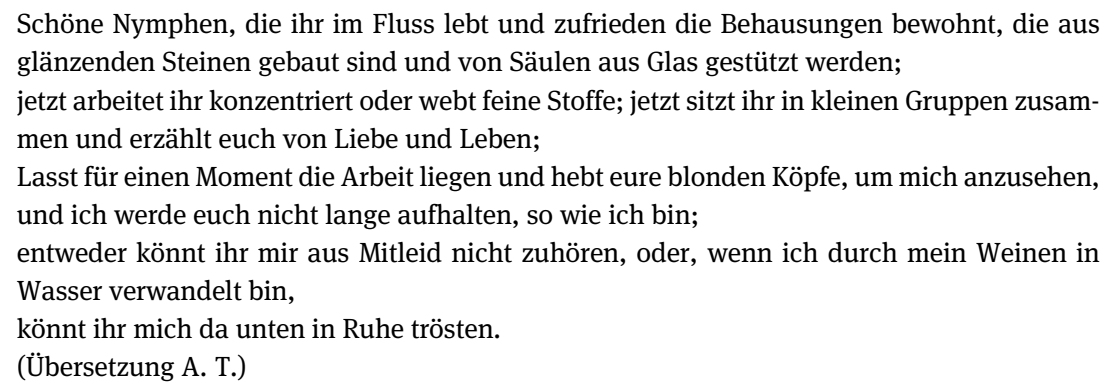

Es sind die ninfas de los ríos, die den Untröstlichen trösten sollen - wenn er sich ihnen, tränennass, ganz anverwandelt hat. Garcilaso hat für die spanische Literatur die Nymphen nachhaltig in den Flüssen platziert; doch schreibt er sich, wie mit seiner Art des Dichtens insgesamt, in lange Traditionsströme ein, neben dem Petrarkismus - der Bezug auf Petrarcas Sonett „Amor che meco al buon tempo ti stavi“ ist bereits von dem zeitgenössischen Kommentator Fernando de Herrera offengelegt worden (303, V. 10-11: „o nimphe, et voi, che'l fresco herboso fondo / del liquido cristallo alberga et pasce“; Herrera 351; Weich 45) - natürlich auch in jenen der Bukolik. Wassernasse Nymphen treffen wir dort in Gestalt der schwimmenden Nymphe Arethusa im 5. Buch von Ovids Metamorphosen, die dann selbst in eine Quelle verwandelt wird; in Giovanni Boccaccios Ninfale Fiesolano (1344/ 1346) ebenso wie in Sannazaros Arcadia (1504) oder, viel später noch, in Honoré d'Urfé's L'Astrée (1607-1621), wo Nymphen nicht allein Seelentrost spenden, sondern den Schäfer Celadon tauchend und tapfer vor dem Ertrinken retten. Der aussergewöhnliche Satz - „esta frase excepcional“ - ist mithin ein vielgesprochener, dessen Echo durch die europäische Literatur hallt - und doch in jedem Text anders klingt.

Einen Echoraum und eine Respondenzinstanz - die kongeniale Landschaft und die Nymphen der Flüsse - habe ich nun als kontextuelle und intertextuelle Dimensionen des Zitats ausgeleuchtet. Die dritte, offensichtlichste, ist die/das Echo selbst: „la dolorosa y húmida Eco“. Húmida, feucht, ist die Versteinerte wohl durch Assoziation mit ihren schwimmenden Schwestern. Dolorosa, schmerzensreich, ist sie in der Tat selbst, ist ihre Geschichte doch die einer unerfüllten Liebe zum selbstverliebten Narziss. Ovid erzählt diese Geschichte im dritten Buch der Metamorphosen und lässt sie so beginnen, dass Echo Opfer eines Fluchs der Juno wird. Durch ihr Redetalent - gegendert und damit pejorativ als Geschwätzigkeit ausgelegt - hatte sie die Göttin hingehalten, damit sie Jupiters Umtriebe mit den anderen Nymphen nicht entdecke. Juno kappt ihr daraufhin das Instrument ihrer Täuschungsfähigkeit, ihre Rede, und lässt ihr nur den „brevissimus usus“ (Ov. Met. III, V. 367): sie kann nur noch die letzten Silben der Rede anderer wie- 
derholen. Das erweist sich als fatal, als Echo sich in Narziss verliebt und sich ihm nicht erklären kann. Sie verzehrt sich vor unerfüllter Liebe und versteinert schließlich, von ihr bleibt nur die Stimme.

Diese Stimme freilich erzeugt mindestens nach frühneuzeitlicher Auffassung keine Kopie des Vorgängigen (s. Traninger, Copia/Kopie). Der Echo wird in der Frühen Neuzeit vielmehr eigene Handlungsmacht oder agency beigelegt, die sie vom Nachhall zum Widerpart werden lässt. Echo antwortet. Quijotes Hoffnung „que le respondiese“ - ist eine, die in der Literatur der Frühen Neuzeit weithin geteilt wird. Bereits das erste Echogedicht der Renaissance, Angelo Polizianos Che fai tu, Eco, aus dem Jahr 1494, inszeniert die Nymphe als (widerständige) Antwortgeberin:

Che fai tu, Eco, mentr'io ti chiamo? - Amo.

Ami tu dua o pur un solo? - Un solo.

Et io te sola e non altri amo - Altri amo.

Dunque non ami tu un solo? - Un solo.

Questo è un dirmi: Io non t'amo - Io non t'amo.

Quel che tu ami ami tu solo? - Solo.

Chi t'ha levata dal mio amore? - Amore.

Che fa quello a chi porti amore? - Ah more!

(225)

Was machst Du, Echo, wenn ich dich rufe?

- Ich liebe.

Liebst Du zwei oder nur einen allein?

- Einen allein.

Und ich liebe Dich allein und keine andere.

- Ich liebe einen anderen.

Dann liebst Du nicht einen allein?

- Einen allein.

Das heißt, Du sagst mir: Ich liebe dich nicht.

- Ich liebe dich nicht.

Der, den Du liebst: liebst Du ihn allein?

- Allein.

Wer hat Dich genommen von meiner Liebe?

- Liebe.

Was macht der, den Du liebst?

- Ah, er stirbt.

(Übersetzung A. T.)

Natürlich ist das vordergründige Prinzip die exakte Wiederholung, doch sind Vertauschung der Ich-Origo und Homophonie wesentliche Mittel des Unterschiebens gegenläufigen Sinns. Echo wiederholt nicht, sie widerspricht - bzw. genauer, sie widerspricht, indem sie wiederholt und bezieht mit vorgegebenen Worten eigene Position. Echo ist in der Frühen Neuzeit durchweg und in einer nicht einmal an- 
zitierbaren Fülle von Text- und Musikbelegen, vom Echogedicht bis zur frühen Oper, „sprachlich-akustisch manifestierte Zwei-Werdung“, wie Jörg Jochen Berns formuliert hat (150; vgl. auch Wald; van Ingen; Hanning; Colby). Von einer, die sich nicht ausdrücken kann, wird sie in der Frühen Neuzeit zu einer, die auf alles eine Antwort weiß, mehr noch, sie wird zum Orakel, zur „Wahr- und Vorsagerin [...] derer Sachen / welche kurtzkünfftig haben geschehen sollen“" (Birken 31). Als Echo beispielsweise in Erasmus' von Rotterdam gleichnamigem Dialog aus dem Jahr 1526 gefragt wird, ob sie die Zukunft vorhersagen kann, antwortet sie sinngemäß: selbstverständlich (555). Das Echo tritt durchweg dem Ich gegenüber und respondiert lautgleich in einer unbotmäßigen und dabei eminent wahrheitsfähigen Weise.

Vor diesem Hintergrund ist es nur folgerichtig, dass es gerade die Echo-Stelle in Cervantes' Quijote ist, an der Borges' Erzähler deutlich Stil und Stimme Menards hört. Ihm widerspricht Echo nicht, vielmehr stützt das simple Faktum ihres modus operandi seine Überzeugung von der Differenz zweier identischer Texte, und mehr noch, der Transformation eines einzelnen durch die Zeit. Menard wollte dezidiert nicht kopieren - er ist nicht einmal ein „peculiar copyist“ (Aguilar 168) -, insofern ist die deviante Echo Emblem und Verkörperung seines Vorhabens. Das Stichwort Echo indiziert alles, was im Rest der Erzählung entfaltet wird: Es geht nicht allein um eine Revision des Konzepts der Lektüre (vgl. Giskin; Prieto; Rabell u.a.) oder gar die Geburt des Lesers, deren Voraussetzung der Tod des Autors sei (Barthes 67). Vielmehr wird darüber hinausgehend das viel stärkere Argument inszeniert, dass der literarische Text die Fähigkeit hat, Bedeutung nicht nur zu transportieren, sondern diese in der Zeit jeweils neu zu stiften. Das Echo macht es vor: Wortgleiches wird nicht nur unterschiedlich gelesen, sondern sagt Unterschiedliches aus - abgesehen davon, dass ,der Text ${ }^{`}$ als stabile Entität immer schon problematisch ist. Immer schon ist er medial und material rekonfiguriert, durch unterschiedlichste Akteure redigiert, korrigiert, layoutiert, annotiert, ediert, gedruckt und vertrieben. Diese Aspekte sind nicht akzidentiell, sondern konstituieren je spezifische Aggregatzustände, die in unterschiedliche Kontexte eingeflochten sind, die ihnen keineswegs gänzlich äußerlich sind. Das Echo, man vergisst es ob seiner Unmittelbarkeit, ist ebenso ein mediales Phänomen in der Zeit. Nachhallen, das ist es, was Texte tun; es ist das Spezifikum der Literatur, dass sie durch die Jahrhunderte Beziehungen herstellt, wiedergelesen, kreativ anverwandelt und wiedervertextet wird. Ein Modell der Literaturgeschichte wird daher gut daran tun, auf eine Theorie der Resonanz zu gründen.

Eine solche Theorie der Resonanz ist nicht allein in den Echo(s) zwischen Cervantes und Borges angelegt, sie lässt sich von dort aus auch mit Blick auf eine wiederholt ins Spiel gebrachte Referenzfigur entwickeln. Es ist dies Paul Valéry, dessen manifeste und latente Präsenz in Pierre Menard durchaus bereits kommen- 
tiert wurde (vgl. z.B. Prieto 54, 69-75; Girardot 70-71; Isava). Die Verneigung vor Valéry ist kein Zufall. Borges war ein fleißiger Valéry-Leser, er zitierte ihn häufig und widmete ihm mindestens fünf seiner Texte (Isava 39). In der Erzählung taucht Valéry als Zeitgenosse und enger Vertrauter Menards auf, in dessen Werkverzeichnis figuriert er mehrfach im Modus der Allusion (Wehr 356-360) und zweimal explizit:

o) Una trasposición en alejandrinos del Cimetière marin, de Paul Valéry (N. R. F., enero de 1928).

p) Una invectiva contra Paul Valéry, en las Hojas para la supresión de la realidad de Jacques Reboul. (Esa invectiva, dicho sea entre paréntesis, es el reverso exacto de su verdadera opinión sobre Valéry. Éste así lo entendió y la amistad antigua de los dos no corrió peligro.) (445)

o) Eine Übertragung des Cimetière Marin von Paul Valéry in Alexandriner (,N. R. F.‘, Januar 1928)

p) Eine Invektive gegen Paul Valéry in den Blättern zur Tilgung der Realität von Jacques Reboul. (Diese Invektive ist, in Klammern gesagt, die genaue Kehrseite seiner wirklichen Meinung über Paul Valéry. Dieser verstand sie auch so, und die alte Freundschaft der beiden kam nicht in Gefahr.) (37)

Menard erscheint hier nachgerade echohaft als Wiederschreiber ebenso wie als Widersprecher: Eine Fassung von Valérys berühmtem Cimetière marin, die dem originalen décasyllabe zwei Silben pro Vers hinzufügt, widersetzt sich der Autorintention. Valéry hatte sich ein strenges rhythmisches Korsett auferlegt und wollte den von ihm als „karg und monoton“ („pauvre et monotone“) beschriebenen Zehnsilbler so nutzen, dass die Verse an Kraft den spätestens seit der Pléiade höhergeschätzten Alexandrinern in nichts nachstünden (,tenter de porter ce Dix à la puissance du Douze“; alle Zitate Valéry, „Au sujet du Cimetière marin“ 1503). Wie eine widerborstige Echo fügt Menard jene zwei Silben hinzu, die ihr zu sagen vergönnt sind.

Das geteilte Verständnis der Invektive als von persönlicher Überzeugung entkoppelte Form, Gegenstand des Eintrags p), ist das Unterpfand einer unerschütterlichen Freundschaft. In der Forschung wurde die Gattungsreferenz kurzgeschlossen mit der Urteilssuspension der Skepsis (Castillo 419) oder aber mit der (vermeintlichen) Praxis des Symbolismus „of saying the opposite of what is meant“ (Woof 225). Im Hinblick auf das untergründige Projekt des Wiederschreibens ist freilich ein anderer Aspekt bedeutender. Es ist dies die Disjunktion von lebensweltlicher Person und rhetorischer persona, präfiguriert diese rhetorische Konvention doch die Konturierung des Erzählers als Zurechnungsinstanz anstelle des Autors (Traninger, „Erzähler und persona“). Hier ist die Entkopplung von Sprechakt und Text verankert, indem die rhetorische oder aber erzählerische Ich-Origo in 
den Text selbst verlagert wird und als Maske (lat. persona) zur Disposition steht. Eintrag p) verweist also nicht allein auf Valérys literarischen Doppelgänger Monsieur Teste (vgl. De Man 58), sondern auf die narratologische Voraussetzung für das Neuschreiben des Quijote insgesamt.

Es ist freilich eine der Allusionen, und zwar prominent im ersten Eintrag des Werkkatalogs, die am deutlichsten auf Paul Valéry als jenen Dialogpartner zeigt, auf den der Blick zu richten ist, wenn es um die literaturtheoretische Relevanz und Resonanz - der Nymphe Echo geht:

a) Un soneto simbolista que apareció dos veces (con variaciones) en la revista La Conque (números de marzo y de octubre de 1899). (444)

a) Ein symbolistisches Sonett, das zweimal (mit Variationen) in der Zeitschrift ,La Conque` (März und Oktober 1899) erschien. (36)

Es war Valéry, der, achtzehnjährig, in der Zeitschrift La Conque erstmals als Dichter in Erscheinung getreten ist - freilich nicht 1899, sondern am 15. März 1891 und mit einem Gedicht, das den Echo-Mythos in sich mitführt: Narcisse parle. Valéry reiht sich damit nicht allein in die ebenso lange wie reiche Tradition des transformierenden Wiederschreibens einer Metamorphose ein, er hat auch selbst Varianten des Gedichts nicht zweimal, sondern zwölfmal an verschiedenen Stellen veröffentlicht (Simmons 85; zu den Fassungen vgl. Euvres I: 1551-1561).

Dem ersten Rezensenten der Fassung von 1891 stach ein Vers besonders ins Auge: Bereits (aber nicht nur) in seinem ersten Narcisse-Gedicht übt sich Valéry im traditionellen Nymphenanruf, nicht ohne diesen freilich neu und gänzlich ungewohnt klingen zu lassen. Einen ein wenig langatmigen und bizarren Singsang („de la mélopée un peu trainante et bizarre“) diagnostiziert Henri Chantavoine in seiner Besprechung im Journal des débats politiques et littéraires vom 7. April 1891 insgesamt, doch hebt er den Vers „Et, vers vous, Nymphes, nymphes, nymphes des fontaines“ (La Conque IV-V) hervor, „dont la plainte prolongée caresse, mais surprend l'oreille“ (Chantavoine o. S.; „dessen sich hinziehende Klage das Ohr liebkost, es aber auch überrascht“). Der Vers, der in anderen Versionen eine einzelne Nymphe anspricht (,Et vers vous, Nymphe, Nymphe, ô Nymphe des fontaines“, Euvres I: 82, V. 3) birgt in sich nicht weniger als ein Echo.

Carolyn Josenhans Simmons hat gezeigt, dass nicht allein, wie allgemein akzeptiert, Narziss ein „emblem for self-reflection and for Valéryan poetics“ (78) ist, sondern dass Valéry der und dem Echo eine durchgängige Präsenz in diesen Gedichten gegeben hat: „Valéry did ascribe great significance to Echo, progressively developing her - particularly in the Narcissus poems - into a figure for the vital, recuperative, and critically-conscious voice of his poetry“ (78). Diese Denkfigur bleibt freilich nicht auf die Narcisses beschränkt. Prominent figuriert sie in der 
achten Strophe von Le Cimetière marin, wo sich das Echo aus der Tiefe des Ichs als Quelle der Dichtung zeigt (Simmons 80):

\author{
O pour moi seul, à moi seul, en moi-même, \\ Auprès d'un cœur, aux sources du poème, \\ Entre le vide et l'événement pur, \\ J'attends l'écho de ma grandeur interne, \\ Amère, sombre et sonore citerne, \\ Sonnant dans l'âme un creux toujours futur! \\ („Le Cimetière marin“, V. 43-48) \\ Für mich allein, in mir, in mich versunken, \\ An einem Herz, am Quell der Dichtkunst trunken, \\ Zwischen der Leere und dem Urgeschehn, \\ Harr' ich des Echos meiner innern Werte, \\ Zisterne, bittre, düstre, klangbeschwerte, \\ Der Seele singend künftiges Vergehn! \\ („Le Cimetière marin“, übers. v. Zidek 109)
}

Simmons sieht Echo als innere Stimme mit Narziss verschmolzen (Simmons 80), doch scheint es genau die alte Vorstellung der Echo als Abspaltung des Ichs (vgl. Kaufmann) zu sein, die die Verse unterfüttert. Doch die Echo, die zwischen Cervantes, Borges und nun auch Valéry mittelt, weist nicht allein auf den schöpferischen Prozess des Dichters, sondern zeigt vielmehr insgesamt auf die historische Tiefendimension der Literatur, um die es im Pierre Menard prominent geht und die Valéry intensiv beschäftigt hat. Valéry kehrte nicht nur zum Narcisse, sondern auch zu den Nymphen immer wieder zurück. 1944 erschien eine „Traduction en vers des Bucoliques de Virgile"; ein weiterer Text, der sich in die bukolische Tradition einreiht, bietet ihm wiederum den Aufhänger für eine explizite Reflexion über das Wiederlesen von Texten - die aber, wie zu zeigen sein wird viel mehr impliziert.

Jean de La Fontaines Kleinepos Adonis - ebenfalls ein mehrfach, nämlich 1658 und 1669, geschriebener Text - liefert Valéry zunächst und vordergründig den Aufhänger für eine Meditation über die Bedeutung der strengen metrischen Form in der Literatur. La Fontaine, der mit seiner meisterhaften Handhabung des Alexandriners Racine vorweggenommen habe, stelle man sich gemeinhin träumerisch und unbekümmert vor, was freilich nichts anderes als ein Effekt seiner metrisch streng gefügten Texte sei. Doch mehr noch: nicht allein Venus und Adonis bewegen sich in diesem literarischen Arkadien (s. Bremond), auch La Fontaine selbst stelle man sich in genau dieser von ihm so meisterhaft evozierten Anderswelt vor: 
Nous le plaçons naturellement dans un parc, ou dans une campagne délicieuse, dont il recherche les belles ombres. Nous lui donnons l'attitude enchantée d'un solitaire qui jamais n'est véritablement seul; soit qu'il se réjouisse avec lui-même de cette paix qui l'environne, soit qu'il cause avec le renard, la fourmi, ou quelque autre de ces animaux du siècle de Louis XIV qui parlaient un si pur langage.

Si les bêtes l'abandonnent [...], il se tourne vers le pays étendu au soleil, où il écoute le roseau, le moulin, les nymphes se répondre. („Au sujet d’Adonis“ 474)

Wir sehen ihn natürlich in einem Park oder einer lieblichen Landschaft, wo er den wohltuenden Schatten sucht. Wir geben ihm die beseligte Heiterkeit eines Einsiedlers, der nie wirklich einsam ist, ob er nun allein mit sich selbst den ihm umgebenden Frieden genießt, oder ob er sich mit dem Fuchs, der Ameise oder einem anderen der Tiere aus dem Jahrhundert Ludwigs XIV. unterhält, die alle eine so reine Sprache sprechen. Überlassen die Tiere ihn dann wieder sich selbst [...], wendet er sich der sonnenbeschienenen Landschaft zu oder lauscht, wie Schilf, Mühle und Nymphen einander antworten. („,Zu ,Adonis‘ von La Fontaine“ 48)

Dass er La Fontaine selbst in die Landschaft der Fiktion versetzt, in der die Nymphen antworten, verweist natürlich zum einen auf die höfischen Divertissementpraktiken - die bergeries oder Schäfereien - des 17. Jahrhunderts, zum anderen aber kommt es Valéry auf ein weiteres Echo an. Seinen Essay „Au sujet d'Adonis“ verfasste er 1920 auf dem südfranzösischen Landgut La Graulet - wir wissen dies, weil es ihm auf diese spezifische Lokalisierung ankam und er sie explizit benannte. Seinem Essay stellte er eine „Note préliminaire“ voran, die, ganz ungewöhnlich, über den spezifischen Schreibort in einer „belle campagne“ reflektiert und diese als ,Idylle“ ausweist („Au sujet d’Adonis“, notes 1739; der Text erschien am 21. Februar 1921 in La Revue de Paris, 540-562). Wenn sich Valéry nun selbst mit dem Verfasser des Adonis synchronisiert, dann tut er dies nicht zufällig vermittels der Evokation einer der am stabilsten codierten poetischen Landschaften. Angesichts der Kontinuität der Lokalitäten - der Tod des Adonis am locus amoenus, der Dichter imaginiert an einem ebensolchen, der Kritiker physisch ebenda mehr als zweihundertsechzig Jahre später - fällt umso deutlicher auf, dass der Text unter der Hand ein anderer geworden ist:

[...] et donc le changement d'époque, qui est un changement de lecteur, est comparable à un changement dans le texte même, changement toujours imprévu, et incalculable. Réjouissons-nous de pouvoir encore lire Adonis, et presque tout avec délices ; mais ne pensons pas que nous lisions celui même des contemporains de l'auteur. Ce qu'ils prisaient le plus, peutêtre nous échappe-t-il ; ce qu'ils regardaient à peine nous touche quelquefois étrangement. Certaines choses charmantes se sont faites profondes ; d'autres, tout insipides. („Au sujet d'Adonis“ 494) 


\begin{abstract}
Daher ist eine Veränderung der Epoche und damit des Lesers einer im Text selbst eingetretenen Veränderung vergleichbar, einer Veränderung, die nie vorausgesehen werden kann und nicht abzuschätzen ist. Freuen wir uns, daß wir Adonis noch immer und fast in allen Teilen mit Entzücken lesen können; aber glauben wir nicht, denselben Adonis zu lesen wie die Zeitgenossen La Fontaines. Was sie am höchsten daran schätzten, entgeht uns vielleicht, was sie fast nicht beachteten, kann uns ganz besonders berühren. Manche charmanten Dinge sind bedeutungsvoll, andere ganz ungenießbar geworden. (,Zu ,Adonis‘ von La Fontaine“ 74)
\end{abstract}

Valéry nutzt die Stabilität des pastoralen Modus, um Wandel zu akzentuieren: Wandel nicht zwischen Texten, sondern in ein und demselben Text. Dabei geht es nicht um divergente Lektüren individueller Leser, sondern darum, dass die Semantik von Worten sich im Gefüge der Diskurse im Verlauf der Zeit zwingend wandelt. Genau deshalb ist Pierre Menard der Schauplatz der Abenteuer des Quijote nur als „la tierra de Carmen durante el siglo de Lepanto y de Lope“ (Borges, „Pierre Menard, autor del Quijote“ 448) vorstellbar - das Signifikat ,Spanien“ ist mehrfach überschrieben und exakt als jene Vorstellung, die Cervantes hatte oder haben konnte, nicht mehr greifbar.

Valéry, der in Hans Robert Jauß’ Einschätzung ,nahezu alles vorwegnahm, was die linguistische Poetik, die literarische Semiotik und der Dekonstruktivismus als letzte Neuheit anpries“ (Jauß, „Ein literarischer Robespierre“ 260), hat eine Poetik radikaler semantischer Offenheit formuliert, der es allerdings weniger auf den Geschmack des Lesers als auf die Konstitution des Textes selbst ankommt - und die sich in griffigen und viel zitierten Maximen niedergeschlagen hat: „[...] un mot est un gouffre sans fond“ („Existence du Symbolisme“ 686), „[m]es vers ont le sens qu'on leur prête“ („Commentaires de Charmes“ 1509) und natürlich:

[O]n n'y insistera jamais assez : il n'y a pas de vrai sens d'un texte. Pas d'autorité de l'auteur. Quoi qu'il ait voulu dire, il a écrit ce qu'il a écrit. Une fois publié, un texte est comme un appareil dont chacun se peut servir à sa guise et selon ses moyens: il n'est pas sûr que le constructeur en use mieux qu'un autre. („Au sujet du Cimetière marin“ 1507)

[M]an wird nie genug darauf hinweisen können: Es gibt keinen wahren Sinn eines Textes. Der Autor hat hier keine Autorität. Was immer er hat sagen wollen: er hat geschrieben, was er geschrieben hat. Einmal publiziert, ist der Text wie eine Apparatur, deren sich jeder auf seine Weise und nach seinen Möglichkeiten bedienen kann: es ist nicht sicher, daß der Erbauer sie besser verwendet als irgendein anderer. (,Betrachtungen zum ,Friedhof am Meer““ 524)

Die Echo(s) zwischen Cervantes und Borges beweisen, dass es nicht nur möglich, sondern zwingend ist, mit identischem Text anderes zu sagen. Dabei ist die wörtliche Wiedergabe gerade kein Garant für semantische Stabilität. Im Gegenteil, die 
wortidente Kopie ist dazu geeignet, uns darüber zu täuschen, dass Borges mit der Geschichte von zwei Texten etwas über einen Text in der Zeit erzählt - und, allgemeiner, etwas darüber, wie literarische Texte durch die Zeit signifizieren. Die Frage der Kopie hat für viele Interpret:innen die Dichotomie von Originalität und Epigonalität als Leitdifferenz der literarischen Wertung in der Moderne aufgerufen (Wehr; vgl. die Debatte zwischen Wreen und Janaway; auch das Referat in Aguilar), doch verstellt sie den Blick auf die damit gleichermaßen angestoßene Meditation über die Resonanzfähigkeit der Literatur selbst. Konzeptuell verankert ist diese in der Figur der Echo, und zwar in ihrer frühneuzeitlichen Konturierung.

Hinsichtlich des Begriffs der Resonanz beziehe ich mich nicht auf Hartmut Rosas in den vergangenen Jahren eminent wirkmächtig gewordene „Soziologie der Weltbeziehung“. Rosa entfaltet eine Theorie des ,guten Lebens“ des Einzelnen im Verhältnis zur Gesellschaft, die stabile Resonanzverhältnisse als Prämisse einer gelingenden Weltbeziehung setzt. Resonanz steht dabei in Opposition zu Entfremdung. Bemerkenswerterweise figuriert bei Rosa wiederum ,Echo“ als kategoriales Gegenteil seines Zentralbegriffs der ,Resonanz‘, denn diese dürfe „weder im wörtlichen noch im übertragenen Sinne mit Echo verwechselt werden: Dem Echo fehlt die eigene Stimme, es tritt gleichsam mechanistisch und ohne Varianz auf; im Echo widerhallt nur das je Eigene, nicht das Antwortende.“ (Rosa 285-286; ebenso 317, 327; außerdem 191, 260). Echo ist hier - das sei nicht kritisiert, nur konstatiert, denn immerhin bewegen wir uns auf dem Feld der Soziologie - auf die moderne Semantik des passiven Widerhalls geschrumpft. Resonanz wird dagegen mit Attributen ausgestattet, für die die historische Echo einstand: „Resonanz, so haben wir schon gesehen, bedeutet nicht Echo, sondern Widerstand.“ (416-417) Doch insgesamt liefert die soziologische Resonanz, die als Antwort auf die ,Steigerungslogik‘ der Moderne und die Krisen der Gegenwart verstanden sein soll, keinen unmittelbar fruchtbaren Anknüpfungspunkt für ein Nachdenken über Literaturgeschichte - oder besser: Literatur in der Geschichte.

Warum insistiere ich so auf dem Echo? Weil die frühneuzeitliche Nymphe, die Wieder- und Widersprechende, Patin steht für eine literaturwissenschaftliche Theorie der Resonanz. Eine solche, freilich ohne Echo und unter Bezugnahme auf physikalische Gesetze und sogar relativitätstheoretische Theoreme, hat bereits in den 1990er Jahren Wai-Chee Dimock vorgelegt. Dimock fasst Resonanz im wörtlichen Sinn als basierend auf dem Hören und Wiederhören eines Textes, ungeachtet der Tatsache, dass die Literatur der Moderne gerade nicht dominant an eine oral-aurale Praxis gebunden ist - ganz anders als jene der Vormoderne, die allerdings bei Dimock nicht explizit in den Blick kommt. Der Fokus auf die Akustik im wörtlichen Sinn erlegt dem Ansatz zugleich eine Beschränkung auf, die gar nicht geboten erscheint, ist doch das, was Dimock über Texte in der Zeit zu sagen hat, nicht spezifisch an Lautlichkeit gebunden. Unter dem Schlagwort eines „diachro- 
nic historicism“ argumentiert Dimock, dass der Begriff der Resonanz den Blick darauf lenke, wie Texte durch die Zeit bedeutsam bleiben - weil sie sich ändern. Ganz abgesehen von allen materialen, editorischen, translatorischen oder philologischen Zurichtungen, die einem Text zuteilwerden müssen, damit er überhaupt durch die Zeit verfügbar bleibt, und die ihren je eigenen Beitrag zu seiner konservierenden Transformation leisten (die bei Dimock freilich keine Rolle spielen), überlebt ein Text, weil er semantisch nicht mit sich selbst identisch bleibt. Jede neue Lektüre verwebt ihn mit neuen Anliegen, Kontexten, Assoziationen. Diese akkumulieren sich, werden aber auch wieder abgeschüttelt: „, human beings [...] bring short-lived meanings to long-lived words“ (1061). Nicht das Zeitlose, Klassische oder Ideale ist es, was Literatur überleben lässt, sondern ihre Resonanzfähigkeit.

Diese ephemeren Bedeutungen sind dem Text konzeptionell nicht äußerlich. Ferdinand de Saussure, der aus anderen Gründen, aber doch wie Dimock vom mündlichen Sprachgebrauch ausging bei der Formulierung seines Begriffs des Zeichens, hat mit seiner schwierigen onomatopoetischen Metapher des ,image acoustique“ (98; „Lautbild“), mit der er die Ausdrucksseite des Zeichens (signifiant) gleichsetzt, darauf insistiert, dass dem Zeichen seine Wahrnehmung immer schon eingeschrieben ist. Ebenso birgt es - mindestens in de Saussures Auffassung - keine direkte Weltrelation, sondern bindet auf der Inhaltsseite einen Vorstellungsinhalt (signifié), der vom jeweiligen Zeichengebrauchenden suppliert werden muss. Ein Primat oder eine Kontrolle eines Kommunikationsteilnehmers konventionell gestünde man sie dem Autor $\mathrm{zu}$ - ist aber im Zeichen nicht verankert. Das Zeichen bindet daher genau genommen eine Wahrnehmung und eine Vorstellung zusammen und ist damit eine kommunikative Relation, die umso lebendiger wird, je (sozial, kulturell, historisch) distanzierter seine Gebrauchenden zueinander stehen. Das Zeichen hat semantische Beweglichkeit grundsätzlich in sich angelegt, und durch die Situationsabstraktheit des literarischen Textes gewinnt diese besondere Virulenz.

Das Literarische bestehe dann in einem bestimmten Aufmerksamkeitsregime, das Texte und Leser:innen immer wieder neu zusammenbringt: „The literary, in other words, is not an attribute resident in a text, but a relation, a form of engagement, between a changing object and a changing recipient“ (Dimock 1064). Literatur ist damit nicht allein der beständige Produktionsstrom neuer Texte, sondern gleichermaßen die beständige Relektüre von Texten, wobei diese sich unter der Hand verwandeln. Dabei kommt es Dimock darauf an, dass Literatur eine „demokratische Institution“ sei - bei aller Problematik einer solchen Extension des Begriffs der Demokratie und der Konstruktion von Literatur als Institution ist damit gemeint, dass eine Theorie der Resonanz anders als eine Theorie der Rezeption nicht auf ideale Leserschaften gepolt ist. Vielmehr: „The literary, it seems, 
comes into being not only through the implied reader (see Iser) but also through the reader not implied, not welcome.“ (1067; vgl. Iser). Eine Theorie der Resonanz trägt daher dem tatsächlichen Gebrauch von Literatur Rechnung, denn jenseits der fachdisziplinär gebändigten Lektürepraxis, die periodisch von Kriteriendebatten begleitet wird, regiert die wilde Hermeneutik. Hier findet Dimock den Ansatzpunkt für eine Bestimmung des Literarischen: „In a provisional definition, the literary might refer to that which resonates for readers past, present, and future.“ (1064)

Auf den ersten Blick scheint eine Theorie der Resonanz durchaus auf einer Linie zu liegen mit Positionen der Rezeptionstheorie aus der zweiten Hälfte des 20. Jahrhunderts. In seiner Abschiedsvorlesung hat Hans Robert Jauß vom Paradox von Pierre Menards Unternehmen als dem „experimentum crucis der Rezeptionstheorie“ (Die Theorie der Rezeption 31) gesprochen: es belege „die Nichtidentität des Wiederholten im zeitlichen Abstand der Wiederholung“ (31). Jauß gibt dem von ihm eingeführten Nexus von Erwartungshorizont und Horizontwandel allerdings als Bedingung einen objektivierbaren, „transsubjektive[n] Horizont des Verstehens“ („Literaturgeschichte als Provokation“ 176) mit, der die Wirkung eines Textes bedinge. Die Offenheit, die von Dimock nun aber unter dem Vorzeichen der Resonanz postuliert wird, geht über Jauß ebenso wie über Umberto Ecos Idee einer opera aperta, bei der der Leser ein ästhetisch komplexes Kunstwerk ,in einem Akt der Kongenialität mit seinem Urheber“ (31) vollendet, oder auch Isers Unbestimmtheits- und Leerstellen, die dem Leser nicht mehr als genau abgezirkelte Einsatzpunkte überantworten, weit hinaus.

Eine Theorie der Resonanz verlässt sich nicht auf die Leerstellen, die ein Leser auffüllen mag und auch nicht auf objektivierbare Horizonte, zu denen er sich zu verhalten habe, sondern kalkuliert damit, dass Texte abseits methodisch gebändigter Fachkontexte, wie sie sich seit dem 19. Jahrhundert konsolidiert haben, radikal zur Disposition stehen. Und sie entbindet die Leserschaft von Kongenialitätsansprüchen und distanziert sie so von der partikularen Praxis der Literaturwissenschaft (die zunehmend als solche kritisch reflektiert wird, vgl. u.a. Felski). Dass Valéry La Fontaine in seinen eigenen Text einziehen ließ, legt eine Schiene zu Michel de Certeaus Theorie des Alltagshandelns, in der er das neigungsgesteuerte ,Bewohnen“ als intuitiven Zugang zu Texten beschreibt: „An die Stelle des Autors tritt eine völlig andere Welt (die des Lesers). Durch diese Mutation wird der Text bewohnbar wie eine Mietwohnung.“(27)

Wenn nun der die penible Katalogisierung der obra visible Menards als „illustrativ für die Verfahren der (positivistischen) Literaturgeschichte [zu] lesen“ ist (Rincón 252; ggf. in Sinn einer Parodie, vgl. Molloy 183), was zeigt dann das unterirdische Werk an? „[D]as, was im ,Innern“ des Dichters vor sich geht?“ (Rincón 252; vgl. auch Prieto 59). Vielleicht deutet das unterirdische Werk gar nicht so sehr 
auf das Innere des Dichters als vielmehr auf eine andere Art von Literaturgeschichte, nämlich auf eine Literaturgeschichte als Beziehungsgeschichte (Japp). Auch wenn es aus dem Innern des Dichters geschöpft wird, ist das subterrane Werk ist nicht Ausdruck seiner Psychologie, sondern steht für den Kommunikationsmodus der Literatur. Die Fakten des Positivismus sind damit schlagartig prekär, wenn die Texte als proteisch hinsichtlich sowohl ihrer Bedeutung als auch ihres Sinns aufgefasst werden. Das ist die subterrane Geschichte der Literatur: dass sie durchweg, auch in der originalitätsfixierten Moderne, ein Wiedersagen, ein Widersprechen und Wiederholen ist. Borges selbst dazu:

„El libro no es un ente incomunicado: es una relación, es un eje de innumerables relaciones. Una literatura difiere de otra, ulterior o anterior, menos por el texto que por la manera de ser leída: si me fuera otorgado leer cualquier página actual - ésta, por ejemplo - como la leerán el año dos mil, yo sabría cómo será la literatura del año dos mil.“ („Nota sobre (hacia) Bernard Shaw“ 218)

Das Buch ist nicht ein unmitteilsames Etwas: es ist eine Beziehung, eine Achse unzähliger Bezüge. Eine Literatur unterscheidet sich von einer anderen, sei diese früher oder später, weniger durch den Text als durch die Leseweise: Wäre mir die Gabe verliehen, irgendeine Seite - diese hier beispielsweise - so zu lesen, wie man sie im Jahr 2000 lesen wird, wüßte ich auch, wie im Jahr 2000 die Literatur beschaffen sein wird. („Anmerkungen zu (gen) Bernard Shaw“ 171)

In dem Sinn ist Gérard Genettes Befund, mit dem er seine Überlegungen zu Pierre Menard abschließt, zu modifizieren: Literatur ist nicht allein ,une réserve de formes qui attendent leur sens“ (446), sondern vielmehr nichts weniger als ,une réserve de textes qui attendent leur sens' - ein Vorrat an Texten, die auf ihren Sinn warten. Echo steht dafür Patin.

\section{Bibliografie}

Aguilar, Jésus. „Can Pierre Menard be the Author of Don Quixote?“ Variaciones Borges 8 (1999): 166-77.

Babel, Reinhard. Translationsfiktionen. Zur Hermeneutik, Poetik und Ethik des Übersetzens. Bielefeld: transcript, 2015.

Barthes, Roland. „La mort de l'auteur.“ Le bruissement de la langue. Paris: Seuil, 1984. 61-67.

Berns, Jörg Jochens. „Die Jagd auf die Nymphe Echo. Künstliche Echoeffekte in Poesie, Musik und Architektur der Frühen Neuzeit.“ Die Jagd auf die Nymphe Echo. Zur Technisierung der Wahrnehmung in der Frühen Neuzeit. Bremen: Edition Lumière, 2011. 139-58.

Birken, Sigmund von. Fortsetzung der Pegnitz-Schäferey [...] durch Foridan. Nürnberg: Endter, 1645.

Borges, Jorge Luis. „Anmerkungen zu (gen) Bernard Shaw.“ Werke. Bd. VII. 170-3. 
Borges, Jorge Luis. „Nota sobre (hacia) Bernard Shaw.“ Obras completas. Bd. II. 217-21. Borges, Jorge Luis. Obras completas. 4 Bde. Hg. Carlos V. Frías. Buenos Aires: Emecé, 1960. Borges, Jorge Luis. „Pierre Menard, autor del Quijote.“ Obras completas. Bd. I. 444-50.

Borges, Jorge Luis. „Pierre Menard, Autor des Quijote“. Werke. Bd. V. 35-45.

Borges, Jorge Luis. Werke in 20 Bänden. Hgg. Gisbert Haefs und Fritz Arnold. Frankfurt a.M.: Fischer Taschenbuch, 1991-1995.

Bremond, Mireille. „L'Adonis de la Fontaine et la tradition.“ Revue d'histoire littéraire de la France 97.2 (1997): 200-218.

Castillo, Jorge Luis. „Pierre Menard and the School of the Skeptics.“ Hispanic Review 71.3 (2003): 415-28.

Certeau, Michel de. Kunst des Handelns. Trans. Ronald Vouillé. Berlin: Merve, 1988.

Cervantes, Miguel de. Der geistvolle Hidalgo Don Quijote von der Mancha. Übers. Susanne Lange. München: dtv, 2016.

Cervantes, Miguel de. El ingenioso hidalgo Don Quijote de la Mancha. Hg. John Jay Allen. Madrid: Cátedra, 2004.

Chantavoine, Henri. „Les très jeunes poètes“ (Rezension des ersten Heftes von La Conque). Journal des débats politiques et littéraires (7. April 1891), gallica.bnf.fr/ark:/12148/ bpt6k4653856/f1.vertical. Letzter Zugriff am 10. Okt. 2020.

Colby, Elbridge. „The Echo-Device in Literature.“ Bulletin of the New York Public Library 23 (1919): 683-713, 783-804.

La Conque 1891-1982. Genf: Slatkine Reprints, 1969.

Curtius, Ernst Robert. Europäische Literatur und lateinisches Mittelalter. Tübingen und Basel: Francke, ${ }^{11} 1993$.

De Man, Paul. „A Modern Master.“ Critical Essays on Jorge Luis Borges. Hg. Jaime Alazraki. Boston, MA: Hall, 1987. 55-62 [Erstveröffentlichung in New York Review of Books, 19. Nov. 1964].

Dimock, Wai-Chee. „A Theory of Resonance.“ PMLA 112.5 (1997): 1060-71.

Dopico Black, Georgina. „Pierre Menard, traductor del Quijote; or Echo’s Echoes.“ Cervantes. Bulletin of the Cervantes Society of America 31.1 (2011): 27-49.

Eco, Umberto. Das offene Kunstwerk. Übers. Günter Memmert. Frankfurt a.M.: Suhrkamp, 1977.

Erasmus von Rotterdam, Desiderius. „Echo.“ Opera Omnia Desiderii Erasmi Roterdami. Bd. I. 7. Amsterdam: North-Holland Publishing Company, 1972. 555-8.

Felski, Rita. Hooked. Art and Attachment. London and Chicago, IL: U of Chicago P, 2020.

Genette, Gérard. „La littérature selon Borges.“ Jorge Luis Borges. Hgg. Dominique de Roux und Jean de Milleret. Paris: L’Herne, 1964. 323-7.

Girardot, Rafael. „Ménard o Mallarmé.“ Cuestiones. México: Fondo de Cultura Económica, 1994. 66-80.

Giskin, Howard. „Borges’ Revisioning of Reading in ,Pierre Menard, Author of the Quixote“.“ Variaciones Borges 19 (2005): 103-23.

Hanning, Barbara Russano. „Powerless Spirit. Echo on the Musical Stage of the Late Renaissance.“ Word, Image, and Song. Hgg. Rebecca Cypess, Beth L. Glixon und Nathan Link. Rochester, NY: U of Rochester P, 2013. 193-218.

Herrera, Fernando de. Anotaciones a la poesía de Garcilaos. Hgg. Inoria Pepe und José María Reyes. Madrid: Cátedra, 2001.

van Ingen, Ferdinand. Echo im 17. Jahrhundert. Ein literarisch-musikalisches Phänomen in der Frühen Neuzeit. Amsterdam: Koninklije Nederlands Akademie van Wetenschappen, 2002. 
Isava, Luis Miguel. „Round Trip: De Valéry a Borges... y de Vuelta.“ Variaciones Borges 29 (2006): 35-59.

Iser, Wolfgang. Der Akt des Lesens. Theorie ästhetischer Wirkung. München: Fink, ${ }^{4} 1994$. Janaway, Christopher. „Borges and Danto: A Reply to Michael Wreen.“ British Journal of Aesthetics 32.1 (1992): 72-6.

Janaway, Christopher. „Two Kinds of Artistic Duplication.“ British Journal of Aesthetics 37.1 (1997): 1-14.

Japp, Uwe. Beziehungssinn. Ein Konzept der Literaturgeschichte. Frankfurt a.M.: Europäische Verlagsanstalt, 1980.

Jauß, Hans Robert. „Ein literarischer Robespierre: Paul Valéry am Beginn einer neuen Rezeption?" Studien zum Epochenwandel der ästhetischen Moderne. Frankfurt a.M.: Suhrkamp, 1989. 257-66.

Jauß, Hans Robert. „Literaturgeschichte als Provokation der Literaturwissenschaft.“ Hans Robert Jauß, Literaturgeschichte als Provokation. Frankfurt a.M.: Suhrkamp, 1970. 144-207.

Jauß, Hans Robert. Die Theorie der Rezeption - Rückschau auf ihre unerkannte Vorgeschichte. Konstanz: Universitätsverlag, 1987.

Kaufmann, Michaela. „The Nymph's Voice as an Acoustic Reflection of the Self.“ The Figure of the Nymph in Early Modern Culture. Hgg. Karl A. E. Enenkel und Anita Traninger. Leiden und Boston: Brill, 2018. 396-418.

La Fontaine, Jean de. „Adonis.“ Fuvres complètes. Bd. 2. Hg. Pierre Clarac. Paris: Gallimard (Bibliothèque de la Pléiade), 1943. 3-19.

Molloy, Sylvia. „Disparate Libraries, Erratic Scribes: Borges and Literary History.“ Romanic Review 100.1-2 (2009): 181-5.

Montemayor, Jorge de. Los siete libros de La Diana. Hg. Asunción Rallo. Madrid: Cátedra, 2008.

Ovid. Metamorphosen. Lateinisch/Deutsch. Übers. Michael von Albrecht. Stuttgart: Reclam, 1994.

Petrarca, Francesco. Canzoniere. Hg. Marco Santagata. Mailand: Mondadori, 1996.

Poliziano, Angelo. Rime. Hg. Natalino Sapegno. Rom: Ediziono dell’Ateneo, ${ }^{2} 1967$.

Prieto, Julio. „Pierre Menard, traductor de Valéry. Entre muertes del autor.“ Variaciones Borges 29 (2010): 53-77.

Rabell, Carmen R. „Lenguaje y escrilectura en Pierre Menard, autor del Quijote.“ Hispanic Journal 13.1 (1992): 27-36.

Rincón, Carlos. „Borges und García Márquez oder: das periphere Zentrum der Postmoderne.“ Postmoderne - globale Differenz. Hgg. Robert Weimann und Hans Ulrich Gumbrecht. Frankfurt a. M.: Suhrkamp, 1992. 246-64.

Rosa, Hartmut. Resonanz: Eine Soziologie der Weltbeziehung. Berlin: Suhrkamp, 2016.

Saussure, Ferdinand de. Cours de linguistique générale. Hgg. Charles Bally und Albert Sechehaye. Paris: Payot, 1971.

Simmons, Carolyn Josenhans. „An Echo in the Text: The Narcissus Poems of Paul Valéry.“ Poetry and Poetics. Hgg. A. Maynor Hardee und Freeman G. Henry. Columbia, SC: U of South Carolina (French Literature Series 18), 1991. 78-86.

Snell, Bruno. „Arkadien. Die Entdeckung einer geistigen Landschaft (1945/1955).“ Europäische Bukolik und Georgik. Hg. Klaus Garber. Darmstadt: Wissenschaftliche Buchgesellschaft, 1976. 14-43.

Stierle, Karlheinz. „Die Entdeckung der Landschaft in Literatur und Malerei der italienischen Renaissance." Vom Wandel des neuzeitlichen Naturbegriffs. Hg. Heinz-Dieter Weber. Konstanz: Universitätsverlag, 1989. 33-52. 
Traninger, Anita. „Erzähler und persona. Rhetorik und Narratologie zwischen Mündlichkeit und Schriftlichkeit.“ Wege moderner Rhetorikforschung. Klassische Fundamente und interdisziplinäre Entwicklung. Hgg. Gert Ueding und Gregor Kalivoda. Berlin u.a.: De Gruyter, 2013. 185-210.

Traninger, Anita. Copia/Kopie. Echoeffekte in der Frühen Neuzeit. Hannover: Wehrhahn, 2020.

Valéry, Paul. „Au sujet d’Adonis.“ CEuvres I. 474-95, notes 1739-40.

Valéry, Paul. „Au sujet du Cimetière marin.“ Euvres I. 1496-507.

Valéry, Paul. „Betrachtungen zum ,Friedhof am Meer،““ Werke I. 512-24.

Valéry, Paul. „Le Cimetière marin.“ Euvres I. 147-51.

Valéry, Paul. „Commentaires de Charmes.“ CEuvres I. 1507-12.

Valéry, Paul. „Existence du Symbolisme.“ Fuvres I. 686-706.

Valéry, Paul. „Narcisse parle.“ Fuvres I. 82-3.

Valéry, Paul. Euvres. 2 Bde. Hg. Jean Hytier. Paris: Gallimard (Bibliothèque de la Pléiade), 1957.

Valéry, Paul. „Traduction en vers des Bucoliques de Virgile.“ Fuvres I. 207-81.

Valéry, Paul. Werke. Frankfurter Ausgabe in 7 Bänden. Hg. Jürgen Schmidt-Radefeldt. Frankfurt a. M.: Insel, 1989.

Valéry, Paul. „Zu ,Adonis“ von La Fontaine.“ Werke III. 48-75.

Vega, Garcilaso de la. Poesías completas. Hg. Elias L. Rivers. Madrid: Castalia, 1996.

Vergil. Bucolica. Hirtengedichte. Studienausgabe. Lateinisch/Deutsch. Übers. Michael von Albrecht. Stuttgart: Reclam, 2001.

Wald, Melanie. „Kanon, Kombinatorik, Echokompositionen. Die musikalische Vermittlung zwischen Himmel und Erde in der Frühen Neuzeit." Musiktheorie 23 (2008): 51-70.

Warning, Rainer. „Petrarcas Tal der Tränen. Poetische Konterdiskursivität im Canzoniere.“ Petrarca-Lektüren. Gedenkschrift für Alfred Noyer-Weidner. Hgg. Klaus W. Hempfer und Gerhard Regn. Stuttgart: Steiner, 2003. 225-46.

Wehr, Christian. „Originalität und Reproduktion. Zur Paradoxierung hermeneutischer und ästhetizistischer Textmodelle in Jorge Luis Borges' Pierre Menard, autor del Quijote." Romanistisches Jahrbuch 51.1 (2000): 351-69.

Weich, Horst. „Schöne Nymphen.“ Mythos - Paradies - Translation: Kulturwissenschaftliche Perspektiven. Hgg. Daniel Graziadei u. a. Bielefeld: transcript, 2018. 41-50.

Woof, William. „Borges, Cervantes \& Quine. Reconciling Existence Assumptions and Fictional Complexities in ,Pierre Menard, Author of Don Quixote‘. “Variaciones Borges 7 (1999): 191230.

Wreen, Michael J. „Don Quixote rides again!“ Romanic Review 86.1 (1995): 141-63.

Wreen, Michael J. „Once is not enough?“ British Journal of Aesthetics 30.2 (1990): 149-58.

Zidek, Egon. „Der Friedhof am Meer. Nach Paul Valéry.“ Zeitschrift für französische Sprache und Literatur 68.1-2 (1958): 108-11.

Anmerkung: Die Forschung für diesen Beitrag wurde ermöglicht durch die Deutsche Forschungsgemeinschaft (DFG) im Rahmen des Exzellenzclusters „Temporal Communities: Doing Literature in a Global Perspective“ (EXC 2020, Projekt-ID 390608380). 